\title{
3D NETWORK ANALYSIS FOR INDOOR SPACE APPLICATIONS
}

\author{
E. Tsiliakou* and E. Dimopoulou \\ School of Rural and Surveying Engineering, National Technical University of Athens, 9 Iroon \\ Polytechneiou str, 15780 Zografou, Greece (eva.tsiliakos@gmail.com; efi@survey.ntua.gr)
}

\section{Commission VI, WG VI/4}

KEY WORDS: indoor space, 3D modeling, network analysis, ArcScene, geoprocessing model, Network Analyst, CityEngine

\begin{abstract}
:
Indoor space differs from outdoor environments, since it is characterized by a higher level of structural complexity, geometry, as well as topological relations. Indoor space can be considered as the most important component in a building's conceptual modelling, on which applications such as indoor navigation, routing or analysis are performed. Therefore, the conceptual meaning of sub spaces or the activities taking place in physical building boundaries (e.g. walls), require the comprehension of the building's indoor hierarchical structure. The scope of this paper is to perform 3D network analysis in a building's interior and is structured as follows: In Section 1 the definition of indoor space is provided and indoor navigation requirements are analysed. Section 2 describes the processes of indoor space modeling, as well as routing applications. In Section 3, a case study is examined involving a 3D building model generated in CityEngine (exterior shell) and ArcScene (interior parts), in which the use of commercially available software tools (ArcGIS, ESRI), in terms of indoor routing and 3D network analysis, are explored. The fundamentals of performing 3D analysis with the ArcGIS Network Analyst extension were tested. Finally a geoprocessing model was presented, which was specifically designed to be used to interactively find the best route in ArcScene. The paper ends with discussion and concluding remarks on section 4.
\end{abstract}

\section{INDOOR SPACE}

\subsection{Indoor Space definition}

In the last decade 3D modeling is increasingly oriented towards indoor space and its physical and digital component parts. Zlatanova et al. (2014) define indoor spaces as artificial constructs developed to support human activities comprising sub-regions that 'are occupied by resources, and agents performing activities subject to varying levels of access', while space subdivision must be compliant with the conceptual models. It is clear that the digital analogous of an indoor space needs to be designed and modelled to support these activities. Consideration and application domains have been researched by Giudice et al. (2010) discussing the nature of 'tasks and application areas that require informatics models of indoor space', appropriate indoor geo-referencing schemes and the best technique for accurate indoor localization and tracking.

\subsection{Indoor requirements}

Indoor space is conceptually described, as complying with geometrical, topological and semantical requirements. The spatial extension of indoor space may be modelled as a 'closed volume, bordered by physical unmovable elements and characterized by a semantic meaning' such as rooms. (Zlatanova et al, 2014). Navigation is probably the most common application area requiring accurate models of indoor space. Correct navigation can be performed considering specific components, such as indoor localisation issues, accurate 3D models representing the space subdivision, 3D algorithms for path computation, guidance (Points of Interest), visualisation and correction of the path (Zlatanova et al., 2013). Brown et al., (2013) completed a thorough study on indoor navigation, listing 15 generic requirements. In another paper,
Isikdag et al. (2013) define conceptual requirements for a building model for navigation purposes. Both studies discussed these requirements in terms of geometry, topology and semantics.

A first requirement is that all indoor environments, containing spaces and building elements (e.g. walls) must comprise rich semantics to support better orientation and guidance.

Indoor spaces must also be described geometrically, clarifying spatial properties of containing spaces or building elements, such as materials or opening directions (e.g. windows or doors). The partition of space is another important aspect for indoor applications. Spaces must be categorized based on pre-defined space types depending on the user, scenario and the tasks to be performed (Brown et al, 2013), while the decomposition of indoor environments into smaller parts and their geo-coding supports better guidance and the generation of correct routes. Furthermore, spatial relationships between the elements (e.g. stairs connecting two floors) must be clear for the derivation of a navigation network (Isikdag et al., 2013). This practically means that different types of indoor environments distinguish levels of connectivity of indoor spaces and must be semantically enriched for the correct vertical or horizontal connection of spaces.

Information on the third dimension of fixed building components (such as walls, stairs) is important for improving navigation. It is imperative though to also consider moveable objects and define their functional states (e.g. door can be 'open' or 'closed') and temporal changes in the building. 


\section{INDOOR SPACE MODELING}

\subsection{Modeling indoors}

Indoor routing may improve by choosing how to model and decompose indoor spaces. Worboys and Walton, (2008) discuss how to model indoor space or indoor objects by using spatial models designed for navigation such as:

-2D Vector Models, which consist of object primitives (polylines, polygons).

-Surface Models (2.5D) that use 2D models to visually simulate 3D space (terrain or surface models).

-Boundary Representation models that represent shapes using limits.

•3D "dumb" solid models using basic 3D geometric forms (prisms, cylinders, sphere) that can have solid volumes added or subtracted from them.

-3D parametric solid modeling allowing 3D objects to be generated with modifiable features.

Zlatanova et al, (2014) on the other hand discuss 2D and 3D approaches for subdividing indoor space. They note that space subdivision for navigation is mainly based on the indoor spaces' geometry and less on semantics especially in 2D approaches. Semantics define mostly buildings' structuring including walls or openings (such as doors or windows) and the partitioning of space, information important for navigation. The authors classify the approaches based on the applied partitioning of space into three categories: non partial or partial subdivision, complete subdivision with the derivation of a network and complete regular subdivision with the derivation of a grid (Zlatanova et al, 2014). Semantics may be used to improve the routes.

The first approach uses the geometry from 2D floor plans to derive navigable spaces. Related work can be found in Liu and Zlatanova, (2011) in Lorenz et al, (2006), in Lamarche \& Donikian, (2004).

Complete subdivision of space with the derivation of a network concerns the subdivision of 2D plans into cells based on criteria such as distances between walls, visibility etc. (Zlatanova et al., 2014). Stoffel et al, (2007), Wallgruen (2005) and Gerarerts (2010) have published their research on these approaches.

Complete subdivision of space with the derivation of regular grid is used to generate graphs from regular grids. 2D floors plans overlap with the grids, which obtain semantics of the corresponding objects on the floor plans. Related work can be found in Afyouni et al, (2012) in Bemmelen et al, (1993), Li et al, (2010).

3D approaches are also grouped into partial subdivision (network), complete subdivision (network) and complete subdivision (grid). The first approach combines 2D plans with 3D building volumes (Thill et al, 2011; Slingsby and Raper, 2008). Complete subdivision of space with the generation of a network, considers volumes and surfaces and is based on Poincare duality. Related work with these approaches can be found in Lee (2004) and Boguslawski (2011).

Complete subdivision of space with the derivation of regular grid uses 3D graphs based on grids to describe and represent a 3D indoor space. Related work can be found in Yuan and Schneider (2010), Bandi and Thalmann (1998) and Andujar et al, (2004). 3D models employed for navigation in 3D approaches are characterized by adequate semantic information compared to semantics in 2D approaches; that is because 3D geometries are more complex than 2D, so it is important to simplify them by incorporating semantics in the models.

\subsection{Routing applications}

Indoor space, analysis, navigation and in general routing applications are nowadays the state-of-the-art. Many researches relate to this issue exploring combinations of novel methodologies and software to manage different aspects of this topic. A number of these studies is selected and described in this section, based on the common background they share with the scope of this paper's application. That is because the routes/ network analysis layers are based on 2D floor plans embedded in 3D spaces. For instance, Kim et al, (2009) suggest a method to build a 2D-3D hybrid data model with less complexity which 'combines the 2D topology constructed from CAD floor plans and the 3D visualization functionality'. In another work Atila et al, (2013) investigate 3D network analysis under different types of constraints by visualizing their data based on CityGML, within a Java based 3D-GIS implementation. Liu and Zlatanova (2011) present an algorithm, which is applied to complex 2D floor plans. Their algorithm 'consists of two-level routing: one to get the routes between rooms, and the other applied to single rooms to acquire the detailed route'.

In another work Thill et al., (2011) use their application 3DCityNet which combines 3D indoor/outdoor space components to perform network-based analysis enabling 'the user to query the least-effort route between any two points in the campus routes network'.

\section{CASE STUDY}

\subsection{Input data}

The scope of this paper is to explore the potentials of tools provided by commercial software (ArcGIS, ESRI) in terms of indoor routing and 3D network analysis. Based on the state-ofthe art, it seems that it is feasible to create a three-dimensional network and perform analysis on it (ArcGIS resources), if the source features for a network dataset has a geometry that includes $\mathrm{z}$-coordinate values. Within this context the fundamentals of performing 3D analysis with the ArcGIS Network Analyst extension were explored and tried. Finally a geoprocessing model was generated, which was specifically designed to be used to interactively find the best route in ArcScene.

The model was tested in National Technical University of Athens, in the newest premises of Lampadarios building of School of Rural and Surveying Engineering. 2D architectural plans of the ground floor, first floor and second floor of the Building (figure 1) were used as input data for the creation and analysis on the 3D network, while the corresponding 3D model designed and textured in CityEngine (ESRI) was used for interactive visualization. This 3D CityEngine model (figure 2) was created via CGA (Computer Generated Architecture) rules (Tsiliakou et al, 2013; Tsiliakou et al, 2014), which is basically an algorithmic process. A CGA rule file contains all the necessary rules, which define the building geometry and finally provide very detailed 3D buildings. 


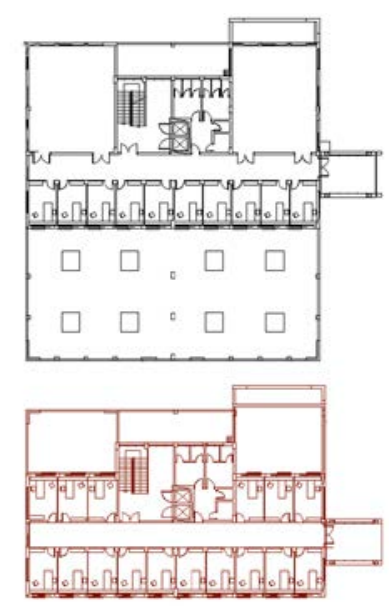

Figure 1. Architectural plans of a part of Lampadarios Building

An example of a rule file (containing all the rules for the creation of a building's geometry) can be seen below.

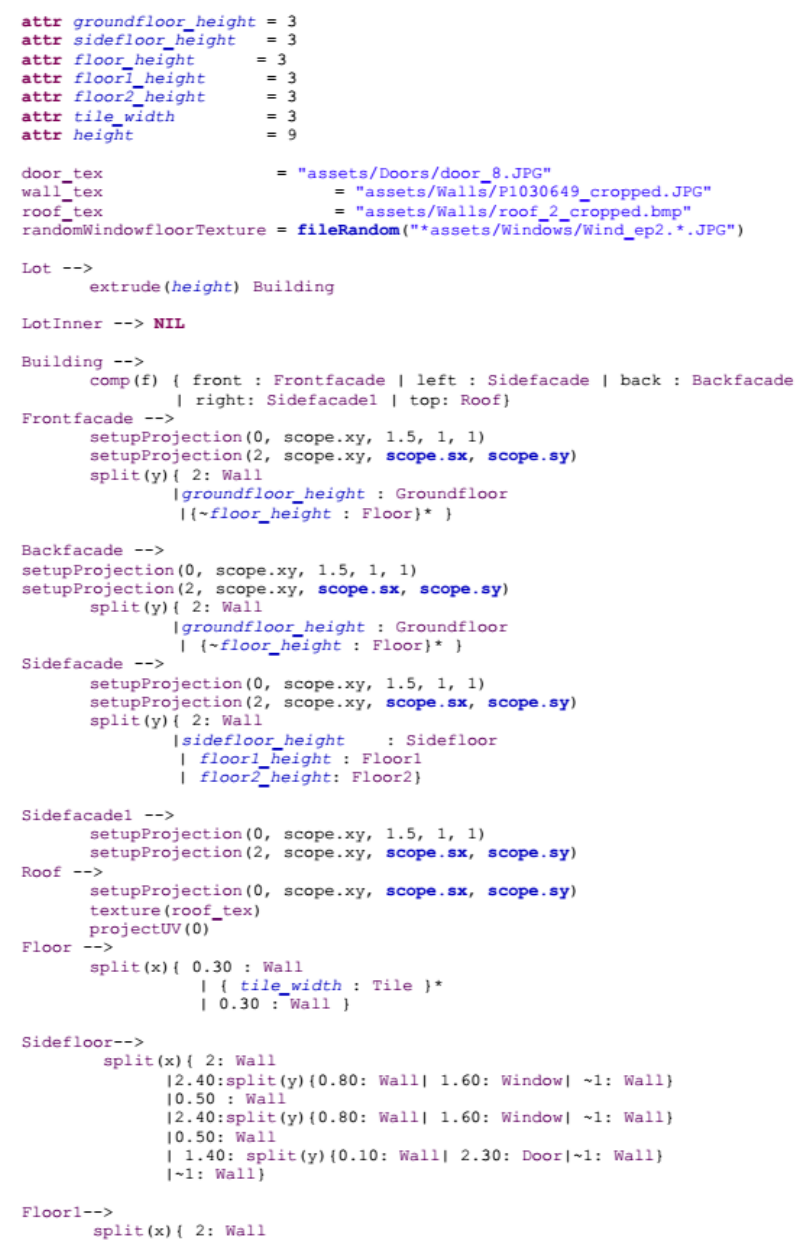

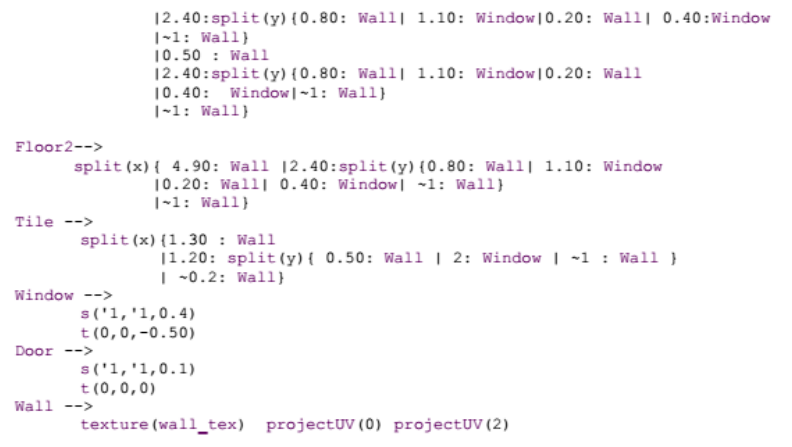

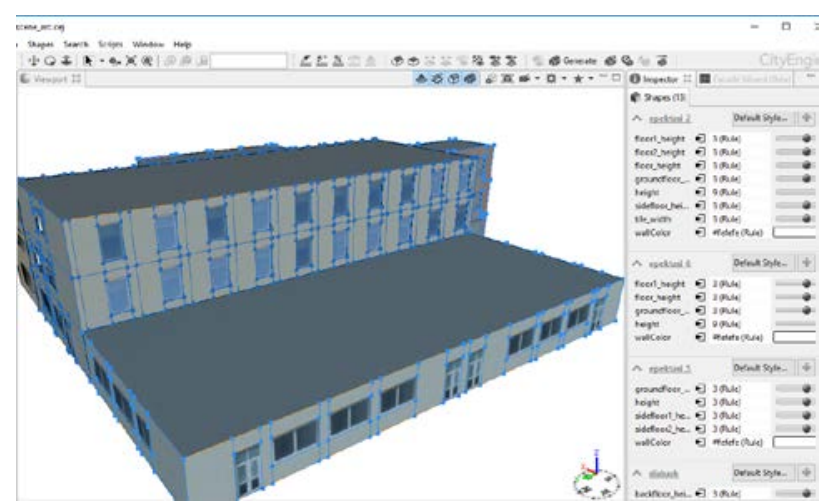

Figure 2. Part of Lampadarios building in CityEngine

\subsection{Geodatabase}

In order to perform routing within a building the data (feature classes) need to be adequately organized in a Geodatabase. The Geodatabase created for this application contains two feature datasets: one including feature classes that define building elements (such as floorplans, walls etc.) and the other including feature classes related to routes within the building.

\subsubsection{Building feature dataset}

The 3D building's interior was created in ArcScene via extrusion, by using as input data the available 2D architectural plans in .dwg format (Autodesk). The initial plans were highly detailed, so a level of abstraction was performed to keep basic elements visualized in the final 3D model. The pre-processed .dwg files were inserted into ArcMap and the feature classes: floorplans, building rooms, walls, doors and the elevator polygon were digitized (figure 3 ). Since the aim of this study is to perform analysis in a 3D building, each feature dataset and class was created by selecting the option 'include z-coordinates' and fields related to elevation and floor number were added, so as to create one feature class containing all floors, etc. The 3D model was built piece by piece in ArcScene (e.g. footprints, walls, doors, stairs) the geometries were extruded at an approximation of 3 meters per floor and the base heights of each floor were calculated based on the field 'elevation' which was predefined as a Geodatabase domain. 


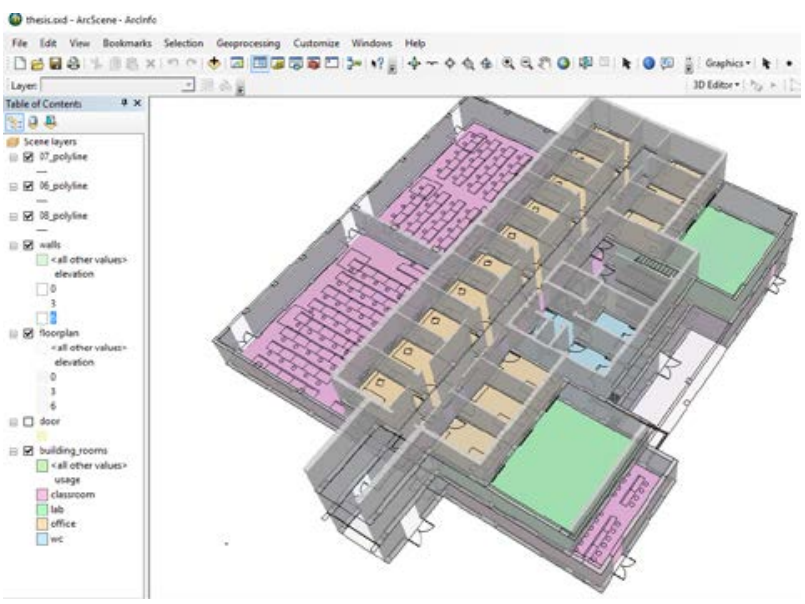

Figure 3. Building's interior in 3D. Different colors apply to different uses

\subsubsection{Routing Dataset}

In order to create a $3 \mathrm{D}$ routing workflow, the shortest route between two points from anywhere within a building had to be calculated. To this purpose, it is necessary to design the basic routes before 'building' the network dataset, to calculate a route. The basic routes are z-enabled and are included in the routing dataset. This dataset comprises feature classes floorlines and floortransitions. Floorlines constitute each floor's routes, while floortransitions comprise 3D elements, such as stairs and elevators.

Rooms and corridors are used as a guide to design z-enabled routes for each floor. This can be completed either in ArcMap or ArcScene. It is necessary to split the routes at intersections, and each point must be correctly snapped both in $2 \mathrm{D}$ and $3 \mathrm{D}$ to the endpoint of a route.

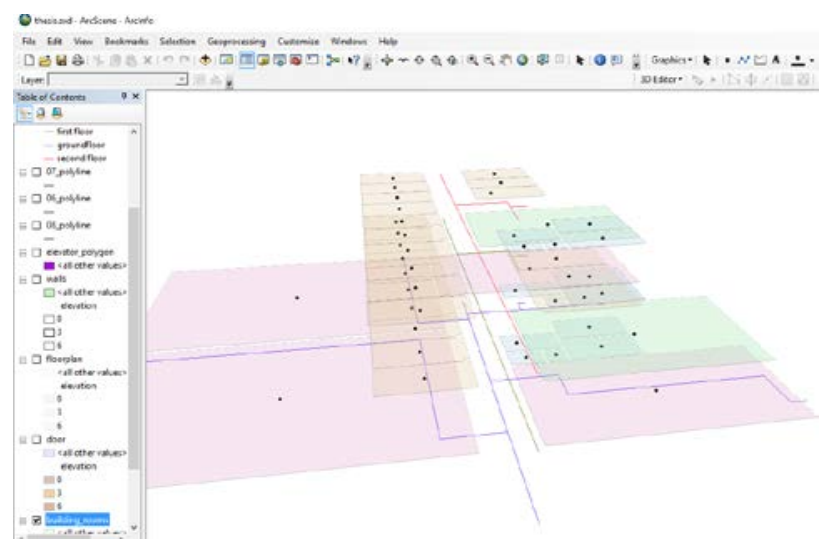

Figure 4. The building's routes in 3D (ArcScene)

The next step is to design the floor transitions in ArcScene. This feature class can only be captured in ArcScene and the capturing process is a challenging task. When designing in 3D, certain requirements must be taken into account. The first one is to define a snapping tolerance such as 0.2 meters (recommended option). The second one is that the corresponding snapping features must be selected (meaning which feature classes to be snapped together). The third one is to select which feature to snap (e.g. endpoint, vertex etc) (in this case endpoint).
If these requirements are fulfilled, 3D elements can be created in ArcScene, otherwise snapping the endpoints of different feature classes together is not feasible. But this is still a challenging task since that creation occurs in xyz space. The optimal way to successfully create a stair or an elevator is to use x,y.z coordinates (either in a local system or at a national grid) as seen in figure 5 . It is vital to snap each point correctly both in 2D and 3D to the endpoint of a route.

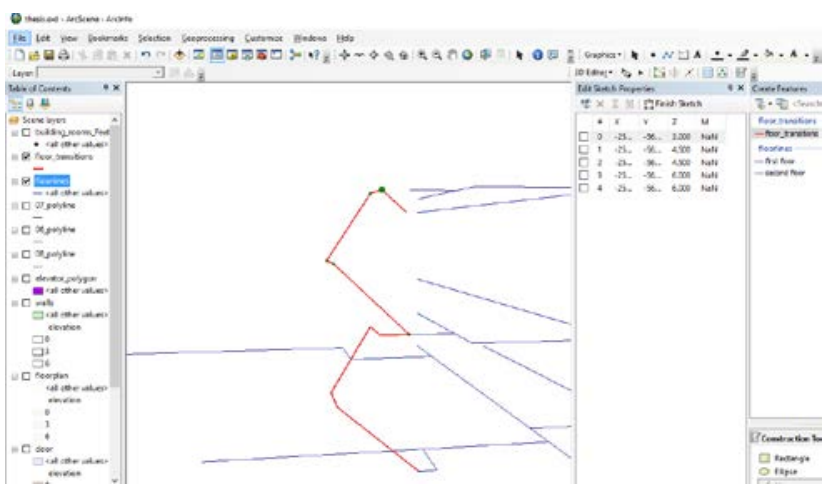

Figure 5. Capturing stairs in ArcScene

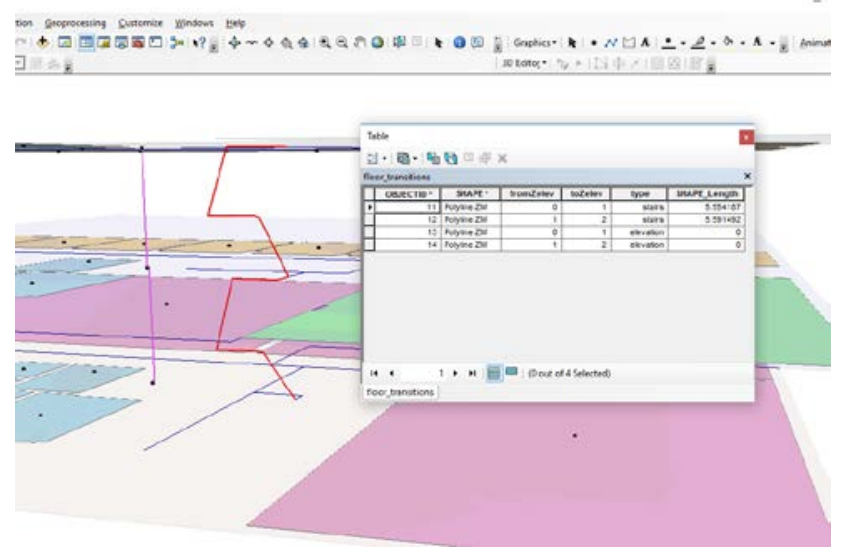

Figure 6. Capturing stairs and elevator in ArcScene (attribute table)

\subsubsection{Network Dataset}

The next step is to create a network dataset from the source feature classes (floor lines and floor transitions). This is similar to creating a 2D network dataset; however the network's elevation is modelled 'Using $\mathrm{Z}$ coordinate values from Geometry'.

There are some main steps when creating a network dataset (ArcGIS resources):

1) All feature classes participating as sources in a network should be present in one feature dataset.

2) The sources (feature classes) must be prepared for certain roles within the network dataset.

3) The sources must have fields representing impedance values - distance, travel time, etc.

The third step is very important, since only shape length was used as an impedance value. Further restrictions such as u-turns, restricted turns or wheelchair routes were not included. Once 
the network dataset is created, network junctions are created as seen in figure 7.

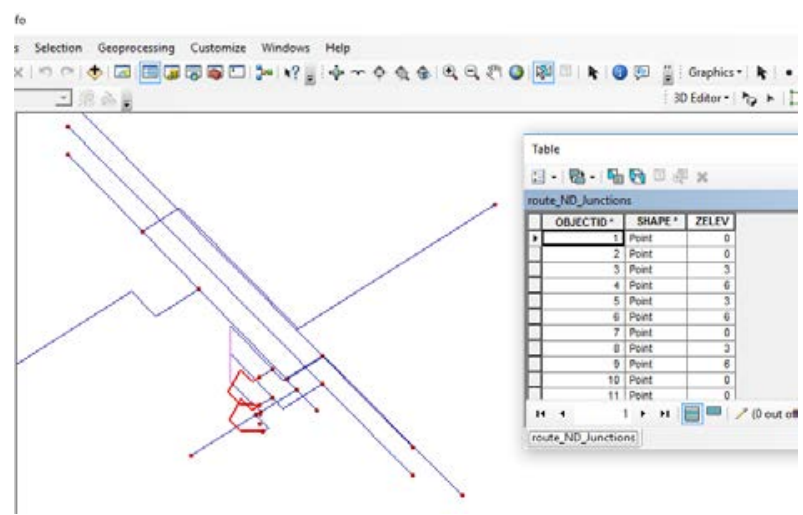

Figure 7. Network junction points

\subsection{Route 3D Model}

Using Model Builder in ArcGIS (figure 8), a geoprocessing model was developed which calculates least-cost routes between stops according to the network dataset in 3D. Stops were interactively added, while the symbology for the model's output network analysis layer is set up in advance. According to ArcGIS resources this can be done in a layer file, which is referenced by the model. The model is comprised by the following elements.

\subsubsection{Make Route Layer}

The Make Route Layer tool creates 'a network analysis layer (Route) that stores the analysis properties, maintains a connection to the input network dataset, and stores the input stops and output routes' (ArcGIS resources). The dataset has a network impedance attribute, which is a parameter.

\subsubsection{Add Locations tool}

The Add Locations tool can be used to add stops manually to the routes (route analysis layers). This is done by clicking on the routes (display screen) in ArcScene. To support the ability of adding stops interactively using the model, 'the Input Locations parameter has to be configured as a feature set' (ArcGIS resources).

The symbology layer file that was previously created, can be used which means that the stops manually added to ArcScene using this model, will be represented by green flags.

\subsubsection{Solve}

The Solve tool computes the least-cost route based on the options specified in the input route analysis layer and creates the routes, which 'are written to the Routes sublayer in the output service area layer' (ArcGIS resources).

\subsubsection{Apply Symbology from Layer}

Apply Symbology takes the symbology from a layer file, including all sublayers, and 'applies it to the route analysis layer created by the model (ArcGIS resources).

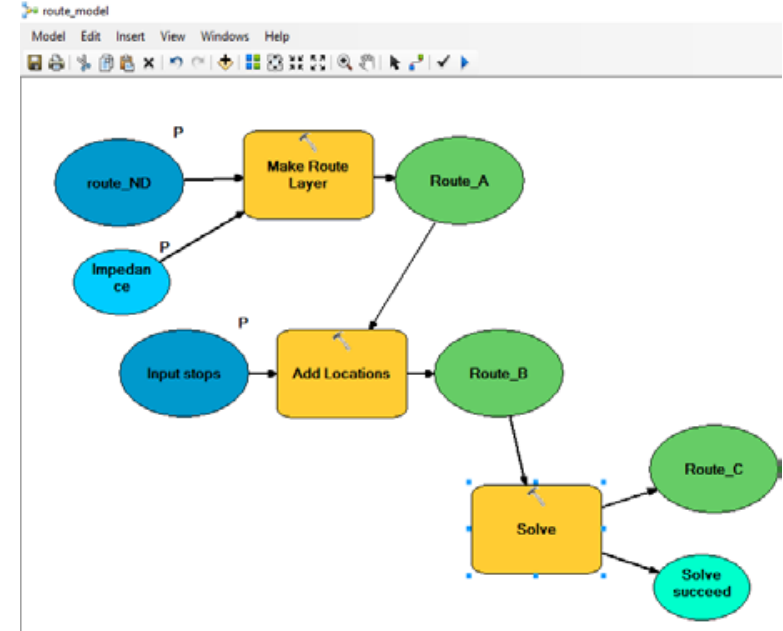

Figure 8. Solve tool

Before using the route model it is important to validate it. Once it is validated it can be then used by clicking on it and selecting input stops on the 3D network. The stops randomly chosen on the network can be named. At last a dialog window will appear defining whether the model has perform analysis successfully\%/or not (figures 9, 10).

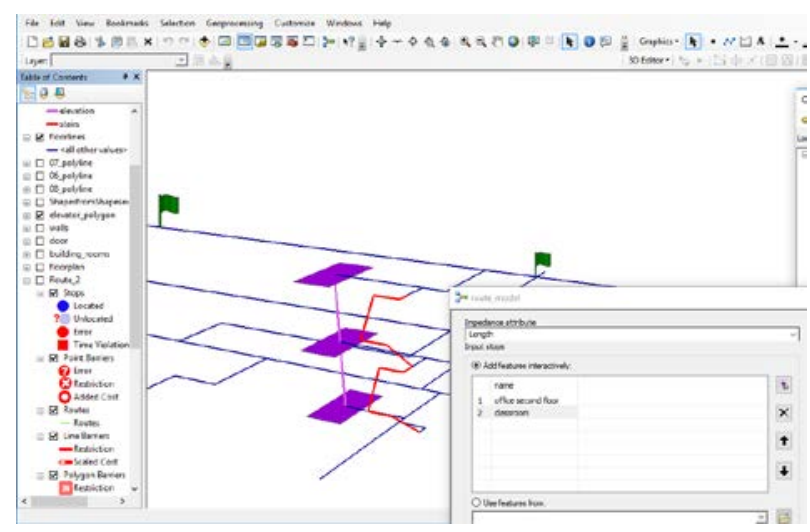

Figure 9. Solve route from a stop in second floor to an exit in ground floor

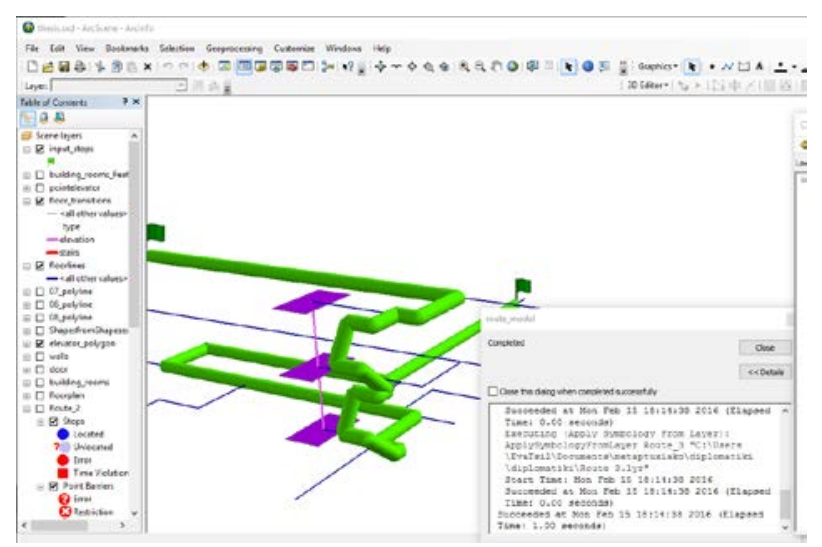

Figure 10. Route succeeded

For better comprehension of the output route, the 3D building model from CityEngine can be used for visualising the exterior shell of the construction. The 3D CityEngine model is exported 
as a file Geodatabase, and can be imported in ArcScene for interactive visualization. This can be achieved by applying transparency on the model, and on the other hand maintaining the successful routes as they are, in order to view them simultaneously (figures 11-13).

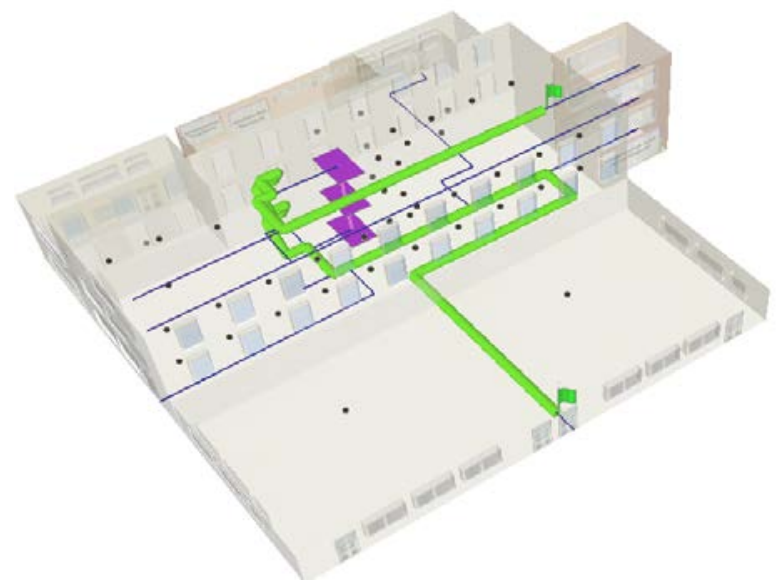

Figure 11. Viewing exterior shell and routes simultaneously

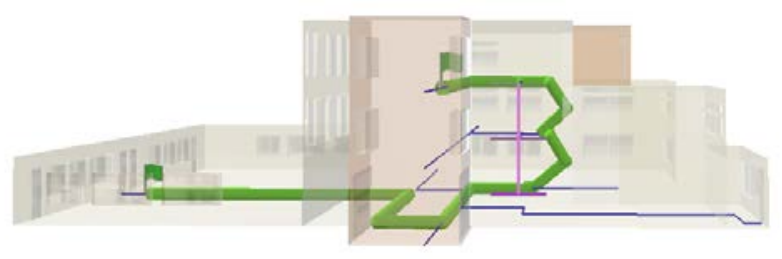

Figure 12. Visualization of the building's exterior shell and the routes simultaneously

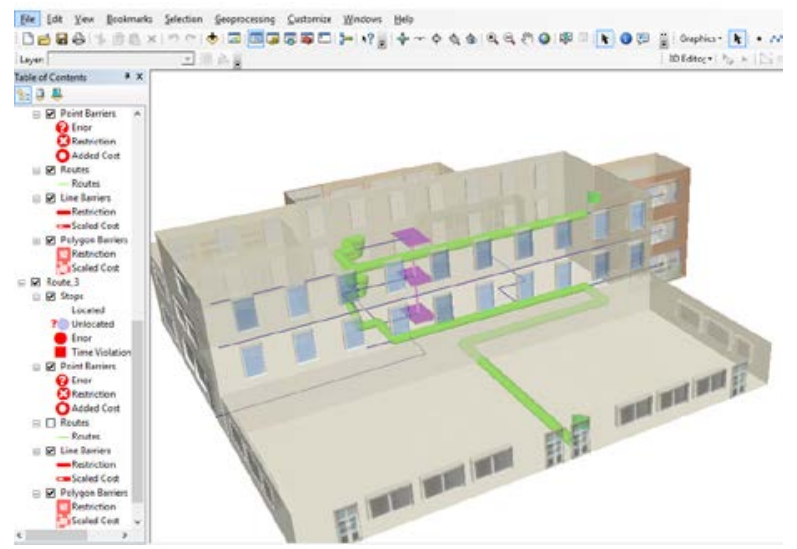

Figure 13. Visualization of the building's exterior shell and the routes

\section{CONCLUSIONS}

The scope of this paper was to explore the possibilities provided by commercial software (ArcGIS, ESRI) in terms of indoor routing. Based on the state-of-the art and the sections analyzed above, it can be easily assumed that if the source features for a network dataset have a geometry that includes $\mathrm{z}$-coordinate values, creating a three-dimensional network and performing analyses on it, might be feasible (ArcGIS resources). Particularly for this paper, the fundamentals of performing 3D analysis with the ArcGIS Network Analyst extension were explored and tested. Finally, a geoprocessing model was presented, specifically designed to be used for interactively finding the best route in ArcScene. The above were tested in the National Technical University of Athens.

The previous chapter's figures give insight on the powerfulness of the geoprocessing tool and the effectiveness of the routing algorithms embedded in ArcGIS Network Analyst. Nevertheless, this insight derives merely from the interactive visualization provided and the effectiveness of the 3D network analysis. In order to evaluate the provided tools and the geoprocessing tool that was implemented, some of the generic requirements listed in the first section need to be considered.

First, all indoor environments, containing spaces and building elements (e.g. walls) must comprise semantics to support better orientation and guidance.

$\checkmark \quad$ The environment built for this paper includes semantics, although not rich such as in the case of a Building Information Model. Specifically, in order to successfully perform network analysis in ArcScene, all data must be organised in a Geodatabase, specifically the feature classes participating in the creation of the network dataset must be part of a distinct dataset within the Geodatabase file.

Semantics relevant to the building components were the definition of floors, building rooms, walls and doors. Extra attribute fields providing more information such as elevation, floor number, the use of a specific room (classroom, lab, office, communal space) and the room's numbering were included.

Semantics relevant to the routing options were definitions of the available routes and the connections between different floors, characterized as floor transitions. Extra attribute fields were included such as the type (stairs, elevators), the transitions (from level, to level), for example from first floor to the second floor and vice versa, and at last elevation and floor number (the last one included only in floorlines).

Another requirement concerns the geometry of indoor spaces. Indoor spaces must be described geometrically, clarifying spatial properties of containing spaces or building elements, such as materials or opening directions (e.g. windows or doors). x The second requirement was taken into account in terms of geometry and visualization. ArcGIS does not explicitly provide available characterizations of the solids created in ArcScene. This means, that the hierarchic relations of building elements cannot be represented such as in a BIM or CityGML. That being said, it is evident that BIM or CityGML are more sufficient tools for representing both semantically and geometrically indoor spaces. The whole building creation process is purely geometrical in ArcGIS.

The partition of space is another important aspect for indoor applications. Spaces must be categorized based on pre-defined space types depending on the user, scenario and the tasks to be performed (Brown et al, 2012), while the decomposition of 
indoor environments into smaller parts and their geo-coding supports better guidance and the generation of correct routes.

$\checkmark \quad$ Theoretically, this is feasible, by adding extra fields in attribute tables (routing feature dataset), such as impedances concerning time, cost, or by adding extra feature classes such as restricted turns, barriers, etc. In this case, the scope was to perform simple routes between two interactively placed points on the model, so length was the only impedance taken into account.

$\checkmark \quad$ The decomposition of space into smaller space was done, by designing the routes (feature class floorlines and floor transitions). This is the efficient way to define subspaces (for routing applications) in ArcScene. These two feature classes formed the basis, on which the network dataset and the network junctions were 'built'.

Furthermore, spatial relationships between the elements (e.g. stairs connecting two floors) must be clear for the derivation of a navigation network (Isikdag et al., 2013). This practically means that different types of indoor environments distinguish levels of connectivity of indoor spaces and must be semantically enriched for the correct vertical or horizontal connection of spaces.

$\checkmark \quad$ These two requirements are taken into account. Specialized types of indoor space and specialized types of connecting space were defined through the routing feature dataset (floor lines and floor transitions). The connection of different sub-spaces such as floors was done via snapping floor transitions to floor lines. This means that spaces are basically represented by linear feature classes, which is the optimal way provided to perform 3D network analysis in ArcGIS. The alternative would be to use polygons or floorplans to automatically derive a network which is done using other (commercial or not) software.

Information on the third dimension of fixed building components (such as walls, stairs etc) is important for improving navigation. It is imperative though to also consider moveable objects and define their functional states (e.g. door can be 'open' or 'closed') and temporal changes in the building. $\mathrm{x}$ These requirements were not taken into account, since the scope of this paper was to perform simple routes between two interactively placed points on the model, so length was the only impedance taken into account. ArcGIS provides the means to add barriers or restrictions to the model, but does not explicitly provide characterizations such as fixed, movable and dynamic obstacle spaces. Concerning semantics defining the surface material and specialist semantics defined for interior and external walls, floors, ceilings, stairs, ramps and general fittings, might be more feasible in a BIM or CityGML, which are more sufficient tools for representing both semantically and geometrically indoor spaces.

Further conclusions concern the creation process of 3D elements with 3D editor in ArcScene. Many users have characterized the $3 \mathrm{D}$ capturing process as challenging, so the snapping features must be refined and be more sophisticated. The snapping problems are also included in the network dataset, which might result in unsuccessfully completed routes when the route model is being used. Nonetheless, ArcGIS provides powerful tools and solutions for finding least-cost routes between two given points on the network dataset in large scale as well. These tools though have to be extended with further attributes.

As mentioned above, this research was oriented to network analysis between two interactively placed points on the model.
Since this work is part of an ongoing project, further possibilities will be explored in order to find the best route between offices or classes by using the non - geometric fields in the attribute tables. It is evident that 3D modeling has taken up the scientific world and technology is moving now rapidly towards providing indoor solutions. Physical and digital components of indoor spaces are explored in detail and novel methodologies, models and/or practises are introduced to the computer scientist community and applied to many aspects of human life on a daily basis.

\section{REFERENCES}

Afyouni, I., Cyril, R. and Christophe, C., 2012. Spatial models for context-aware indoor navigation systems: A survey. Journal of Spatial Information Science, 1(4), pp.85-123.

Atila, U., Karas, I.R. and Rahman, A.A., 2013. A 3D-GIS implementation for realizing 3D network analysis and routing simulation for evacuation purpose. In Progress and New Trends in 3D Geoinformation Sciences (pp. 249-260). Springer Berlin Heidelberg.

Brown, G., Nagel, C., Zlatanova, S. and Kolbe, T.H., 2013. Modelling 3D topographic space against indoor navigation requirements. In Progress and New Trends in $3 D$ Geoinformation Sciences(pp. 1-22). Springer Berlin Heidelberg.

Geraerts, R., 2010, May. Planning short paths with clearance using explicit corridors. In Robotics and Automation (ICRA), 2010 IEEE International Conference on (pp. 1997-2004). IEEE.

Isikdag, U., Zlatanova, S. and Underwood, J., 2013. A BIMOriented Model for supporting indoor navigation requirements. Computers, Environment and Urban Systems, 41, pp.112-123.

Kim, H., Jun, C. and Yi, H., 2009, May. A SDBMS-based 2D3D hybrid model for indoor routing. In Mobile Data Management: Systems, Services and Middleware, 2009. MDM'09. Tenth International Conference on (pp. 726-730). IEEE.

Lamarche, F. and Donikian, S., 2004, September. Crowd of virtual humans: a new approach for real time navigation in complex and structured environments. In Computer Graphics Forum (Vol. 23, No. 3, pp. 509-518). Blackwell Publishing, Inc.

Lee, J., 2004. A spatial access-oriented implementation of a 3-D GIS topological data model for urban entities. GeoInformatica, 8(3), pp.237-264.

Liu, L. and Zlatanova, S., 2011, May. A" door-to-door" Pathfinding Approach for Indoor Navigation. In Proceedings Gi4DM 2011: GeoInformation for Disaster Management, Antalya, Turkey, 3-8 May 2011. International Society for Photogrammetry and Remote Sensing (ISPRS).

Lorenz, B., Ohlbach, H.J. and Stoffel, E.P., 2006. A hybrid spatial model for representing indoor environments. In Web and wireless geographical information systems (pp. 102-112). Springer Berlin Heidelberg.

Stoffel, E.P., Lorenz, B. and Ohlbach, H.J., 2007. Towards a semantic spatial model for pedestrian indoor navigation. In Advances in conceptual modeling-foundations and applications (pp. 328-337). Springer Berlin Heidelberg. 
Thill, J.C., Dao, T.H.D. and Zhou, Y., 2011. Traveling in the three-dimensional city: applications in route planning, accessibility assessment, location analysis and beyond. Journal of Transport Geography, 19(3), pp.405-421.

Tsiliakou, E., Labropoulos, T. and Dimopoulou, E., 2013, November. Transforming 2D cadastral data into a dynamic smart 3D model. In Int Arch Photogrammetry, Remote Sensing Spat Inf Sci, ISPRS 8th 3DGeoInfo conference and WG II/2 workshop (Vol. 2, p. W2).

Tsiliakou, E., Labropoulos, T. and Dimopoulou, E., 2014. Procedural modeling in 3D GIS environment. International Journal of 3-D Information Modeling (IJ3DIM), 3(3), pp.17-34.

Wallgrün, J.O., 2004. Autonomous construction of hierarchical voronoi-based route graph representations. In Spatial Cognition IV. Reasoning, Action, Interaction (pp. 413-433). Springer Berlin Heidelberg.

Worboys, M., \& Walton, L. (2008). Towards a model of indoor space. Maine: University of Maine, 2008.

Yuan, W. and Schneider, M., 2010. iNav: An indoor navigation model supporting length-dependent optimal routing. In Geospatial thinking (pp. 299-313). Springer Berlin Heidelberg.

Zlatanova, S., Sithole, G., Nakagawa, M. and Zhu, Q., 2013, December. Problems in indoor mapping and modelling. In Acquisition and Modelling of Indoor and Enclosed Environments 2013, Cape Town, South Africa, 11-13 December 2013, ISPRS Archives Volume XL-4/W4, 2013. International Society for Photogrammetry and Remote Sensing (ISPRS)

Zlatanova, S., Liu, L., Sithole, G., Zhao, J. and Mortari, F., 2014. Space subdivision for indoor applications. Delft University of Technology, OTB Research Institute for the Built Environment.

ArcGIS resources, last accessed, 27/02/2016 http://desktop.arcgis.com/en/arcmap/10.3/guidebooks/extension s/network-analyst/analysis-on-3d-network-datasets.htm\#GUID2A8CE661-D02B-4F07-99C1-5CD424B8002B 